\title{
Matched-pair analysis of survival in the patients with T3 laryngeal squamous cell carcinoma treated with supracricoid partial laryngectomy or total laryngectomy
}

This article was published in the following Dove Press journal: OncoTargets and Therapy

\author{
Xin Xia ${ }^{1, *}$ \\ Ying-Ying Zhu',* \\ Wen-Wen Diao' \\ Xiao-Li Zhu' \\ Xiao-Hua Shi ${ }^{2}$ \\ Wu-Yi Li' \\ Zhi-Qiang Gao' \\ Guo-Jun $\mathrm{Li}^{\prime}$ \\ Xing-Ming Chen' \\ 'Department of Otolaryngology- \\ Head and Neck Surgery, Peking Union \\ Medical College Hospital, Peking \\ Union Medical College and Chinese \\ Academy of Medical Sciences, Beijing, \\ China; ${ }^{2}$ Department of Pathology, \\ Peking Union Medical College \\ Hospital, Peking Union Medical \\ College and Chinese Academy of \\ Medical Sciences, Beijing, China \\ *These authors contributed equally \\ to this work
}

\begin{abstract}
Background: The survival outcomes between supracricoid partial laryngectomy (SCPL) and total laryngectomy (TL) were compared in the groups of matched-pair patients with T3 laryngeal squamous cell carcinoma (LSCC).

Methods: Patients with T3 LSCC were matched based on prognostic factors. The Kaplan-Meier curve and the Cox proportional hazards model were used for analysis on survival.

Results: A total of 212 patients with T3 LSCC were included after matching (106 underwent SCPL and 106 underwent TL). Multivariable analysis showed no differences in overall survival (hazard risk $[\mathrm{HR}]=1.15 ; 95 \% \mathrm{CI}$ : $0.79-1.67 ; P=0.47)$, disease-specific survival $(\mathrm{HR}=1.11$; 95\% CI: $0.69-1.80 ; P=0.66$ ), and recurrence-free survival (HR=1.07; 95\% CI: $0.68-1.68$; $P=0.77$ ) between the SCPL group and TL group.
\end{abstract}

Conclusion: SCPL provides reliable therapeutic outcomes and can be used to avoid a TL surgery in some patients with advanced primary laryngeal cancer.

Keywords: survival analysis, matched-pair analysis, laryngeal neoplasms, laryngectomy, squamous cell carcinoma

\section{Introduction}

Laryngeal cancer is a relatively common malignant tumor occurring in the head and neck regions and is responsible for $\sim 1 \%$ of all cancer-related deaths. ${ }^{1}$ A study by the National Central Cancer Registry of China showed that $\sim 26,400$ new laryngeal cancer cases occurred and resulted in 14,500 cancer-related deaths in China in 2015, and its incidence and mortality have been increasing in recent years. ${ }^{2}$ Approximately $60 \%$ of the laryngeal cancer patients presented with advanced (stage III or IV) disease at diagnosis. ${ }^{3}$ These patients typically suffered from dysphonia, dyspnea, and even dysphagia, which might cause serious psychological disorders and severely affect patient's quality of life. ${ }^{4}$

Currently, for most of the T3 laryngeal squamous cell carcinoma (LSCC) patients, chemoradiotherapy/radiotherapy or partial laryngectomy might be options for the preservation of laryngeal function, but most of these patients undergo total laryngectomy (TL) for various reasons. TL procedure, however, is not the best therapeutic choice in that patients' speech and physiological airway cannot be preserved due to the loss of organ, and it also causes great pain and inconvenience. Therefore, supracricoid partial laryngectomy (SCPL) is an alternative choice for some patients 
with advanced laryngeal cancer to avoid the TL surgery and its side effects. ${ }^{5-7}$ Nevertheless, in the clinical practice, it is difficult for a surgeon to balance the benefit and risk between complete excision of the lesion and preservation of laryngeal function.

In this study, therefore, we performed a matched-pair analysis in the patients with T3 LSCC to compare survival outcomes of SCPL and TL surgical procedures. To our knowledge, this is the first study to perform a matchedpair design controlling for variables of known prognostic significance.

\section{Patients and methods}

\section{Patient population}

A total of 877 patients with newly diagnosed, pathologically confirmed, and untreated T3 LSCC were enrolled into this study at the Peking Union Medical College Hospital (PUMCH) from January 1995 to December 2011. Epidemiologic and clinical information including age, sex, comorbidity score, tobacco and alcohol consumption, tumor site, overall stage, nodal stage, adjuvant treatment and grade of differentiation were obtained from the participants for this matchedpair analysis. TNM stages were identified in all participants following the TNM classification criteria designated by the American Joint Committee on Cancer (AJCC) in 2010. For the purpose of matched-pair analysis, only the patients who were eligible for either SCPL or TL were enrolled into this study in order to compare the clinical outcomes of these two surgical procedures.

"Smokers" were defined as those who had smoked $>100$ cigarettes in their lifetime, whereas "non-smokers" had smoked 100 cigarettes or fewer. "Drinkers" were defined as patients who drank alcoholic beverages at least once a week for 1 year or longer; otherwise, they were defined as "non-drinkers". 8 A signed written informed consent form for this study was obtained from each participant, and the study protocol was approved by the ethics committee of the PUMCH.

The inclusion criteria were as follows: 1) newly diagnosed, previously untreated, and pathologically confirmed LSCCs; 2) T3 stage originating at anterior two-thirds of the larynx with true vocal cord fixation and/or paraglottic/ preepiglottic space invasion without arytenoid cartilage fixation and cricoid cartilage invasion, which were eligible for either treatment modalities; and 3) patients without resection margins, perineural invasion, or extracapsular nodal spread. The exclusion criteria were as follows: 1) cases had no clear medical documentation or were lost during follow-up;
2) cases had a history of other tumors or tumor-related diseases; and 3) cases had distant metastasis.

\section{Matching criteria}

In the current cohort study, one-to-one match was paired for the patients treated with SCPL or TL surgical procedures. The matching variables were influencing factors including age ( \pm 5 years), gender (male or female), smoking status (nonsmoker or smoker), site of primary tumor (supraglottis or glottis), disease stage (stage III or stage IV), and adjuvant treatment (untreated or radiation treated). Unmatched variables included comorbidity score (none and mild or moderate and severe), alcohol status (nondrinker or drinker), nodal classification (N0, N1, N2, or N3), and grade of differentiation (highly differentiated, moderately differentiated, or poorly differentiated). By the definition of aforementioned inclusion and exclusion criteria, total 106 pairs of (212) patients with T3 LSCC treated with SCPL or TL were finally included in this matched-pair analysis.

\section{Surgical procedures}

The surgical operations were performed according to the original description of the techniques in the literature. ${ }^{5,9}$ The SCPL was classified according to the type of reconstruction: cricohyoidoepiglottopexy (CHEP) and cricohyoidopexy (CHP). It included the removal of the whole thyroid cartilage, both true and false cords, the ventricles, the epiglottis, and the paraglottic and preepiglottic spaces, meanwhile, sparing only the cricoid cartilage, hyoid bone, and at least one functional and mobile arytenoid. ${ }^{10}$

Selective neck dissections were performed in the cases with clinically positive nodules. Elective dissections were performed in $\mathrm{cN} 0$ of the cases with nodular metastasis, with removal of levels II-IV, sometimes including level V, according to the international guidelines. All surgical procedures were performed by the same team of surgeons to ensure comparability between the treatment groups.

\section{Patient's follow-up}

The primary end point was overall survival (OS), defined as the time from the date of starting treatment to the date of death from any cause or last follow-up date. Secondary end points were disease-specific survival (DSS), defined as the time from the date of starting treatment to the date of death from disease or last follow-up, and recurrence-free survival (RFS), defined as the time from the date of starting treatment to the date of recurrence or date of last follow-up. Patients were followed up from the date of surgery with 
regularly scheduled clinical and radiographic examinations. Patients were considered alive and free of disease recurrence if disease absence was documented on the date of the last visit in December 2016. All patients were followed up for a minimum of 5 years or until death.

\section{Statistical analyses}

Data were analyzed using SPSS (SPSS for Windows version 21.0; IBM Corporation, Armonk, NY, USA). Differences between the SCPL and TL groups in DSS, RFS, and OS were compared using the Kaplan-Meier method and the log-rank test for equality of survival curves. The multivariate analysis was performed using the Cox proportional hazards model. Factors that were not matched (comorbidity score, alcohol consumption, nodal classification, and grade of differentiation) were evaluated using the Pearson chi-squared test and the two-tailed Fisher's exact test to detect any significant differences between the SCPL and TL groups. All statistical tests were two tailed, and $P$-values $<0.05$ were considered as statistically significant.

\section{Results \\ Demographics}

Characteristics of the two matched groups are presented in Table 1. The patients were matched by age, sex, smoking history, site of primary tumor, disease stage, and adjuvant treatment. As expected, there were no significant differences between the two groups in terms of the matching variables. Furthermore, we compared the differences in survival by each characteristic, observing significant differences in age, smoking status, tumor stage, adjuvant treatment, and histopathologic grade $(P<0.05$ for each).

\section{Comparison of survival rate}

Patients were followed up at least for a minimum of 5 years or until death. The follow-up time ranged from 6 to 184 months,

Table I Matched, demographic, and exposure characteristics

\begin{tabular}{|c|c|c|c|c|c|}
\hline \multirow[t]{2}{*}{ Variable } & \multicolumn{2}{|l|}{ SCPL $(n=106)$} & \multicolumn{2}{|l|}{ TL $(n=106)$} & \multirow[t]{2}{*}{ Log-rank P-value } \\
\hline & No of patients & $\%$ & No of patients & $\%$ & \\
\hline Age*, years & & \multirow{2}{*}{\multicolumn{2}{|c|}{59.9}} & 0.009 \\
\hline Mean & \multicolumn{2}{|c|}{58.7} & & & \\
\hline Median & \multicolumn{2}{|c|}{57} & \multicolumn{2}{|c|}{61} & \\
\hline Range & \multicolumn{2}{|c|}{$34-70$} & \multicolumn{2}{|c|}{$32-75$} & \\
\hline Sex* & & & & 0.571 \\
\hline Male & 96 & 90.6 & 96 & 90.6 & \\
\hline Female & 10 & 9.4 & 10 & 9.4 & \\
\hline Adult comorbidity score & & & & & 0.558 \\
\hline None and mild & 100 & 94.3 & 94 & 88.7 & \\
\hline Moderate and severe & 6 & 5.7 & 12 & 11.3 & \\
\hline Smoking status* & & & & & 0.021 \\
\hline Smokers & 87 & 82.1 & 87 & 82.1 & \\
\hline Nonsmokers & 19 & 17.9 & 19 & 17.9 & \\
\hline Alcohol & & & & & 0.516 \\
\hline Drinkers & 82 & 77.4 & 88 & 83.0 & \\
\hline Non-drinkers & 24 & 22.6 & 18 & 17.0 & \\
\hline Tumor site* & & & & & 0.766 \\
\hline Supraglottis & 41 & 38.7 & 41 & 38.7 & \\
\hline Glottis & 65 & 61.3 & 65 & 61.3 & \\
\hline Stage* & & & & & $<0.001$ \\
\hline III & 57 & 53.8 & 57 & 53.8 & \\
\hline IV & 49 & 46.2 & 49 & 46.2 & \\
\hline Adjuvant treatment* & & & & & $<0.001$ \\
\hline Untreated & 57 & 53.8 & 57 & 53.8 & \\
\hline Radiation & 49 & 46.2 & 49 & 46.2 & \\
\hline Grade & & & & & $<0.001$ \\
\hline Highly differentiated & 23 & 21.7 & 19 & 17.9 & \\
\hline Moderately differentiated & 65 & 61.3 & 67 & 63.2 & \\
\hline Poorly differentiated & 18 & 17.0 & 20 & 18.9 & \\
\hline
\end{tabular}

Note: *Matched variables: age, sex, smoking status, tumor site, stage, and adjuvant treatment. Abbreviations: SCPL, supracricoid partial laryngectomy; TL, total laryngectomy. 
Table 2 Follow-up outcomes in the two groups

\begin{tabular}{l|l|l|l|l}
\hline \multirow{2}{*}{ Vital status } & \multicolumn{2}{|l|}{ SCPL $(\mathbf{n}=\mathbf{1 0 6})$} & \multicolumn{2}{l}{ TL (n=1 06) } \\
\cline { 2 - 5 } & $\mathbf{n}$ & $\%$ & $\mathbf{n}$ & $\%$ \\
\hline Death, all causes & & & & \\
$\quad$ No & 54 & 50.9 & 45 & 42.5 \\
$\quad$ Yes & 52 & 49.1 & 61 & 57.5 \\
Death, due to a disease & & & & \\
No & 75 & 70.8 & 69 & 65.1 \\
Yes & 31 & 29.2 & 37 & 34.9 \\
Recurrence & & & & \\
No & 69 & 65.1 & 64 & 60.4 \\
Yes & 37 & 34.9 & 42 & 39.6 \\
\hline
\end{tabular}

Abbreviations: SCPL, supracricoid partial laryngectomy; TL, total laryngectomy.

with an average of 72.6 months (median, 69.5 months) for the SCPL group and 84.1 months (median, 77.0 months) for the TL group.

Vital status for the patients of the two groups is listed in Table 2. For the SCPL group, 52 out of the 106 patients died from varying causes, 31 died from certain diseases, and 37 had recurrence. For the TL group, 61 out of the 106 patients died from varying causes, 37 died from certain diseases, and 42 had tumor recurrence. The 5 -year OS rates were $65.8 \%$ and $69.8 \%$ in the SCPL and TL groups, respectively, with no significant difference between the two groups $(P=0.41)$. Similar results were found for DSS (73.6\% vs $77.2 \%$, respectively, at 5 years; $P=0.43$ ) and RFS ( $72.1 \%$ vs $71.6 \%$, respectively, at 5 years; $P=0.66$ ) between the two groups. Furthermore, curves of OS, DSS, and RFS in the SCPL and TL groups are shown in Figures 1-3, respectively. None of OS, DSS, or RFS was significantly different between the SCPL and TL groups ( $P=0.47, P=0.66$, and $P=0.77$, respectively).

\section{Matched-pair analysis}

If a pair of the patients experienced the same events, they were classified as concordant; in contrast, if one patient of a pair experienced an event but the other one did not, they were considered as discordant. ${ }^{11}$ By this definition, in this study, there were 39 concordant pairs in which both the SCPL and TL patients died from all kinds of causes; in the 13 discordant pairs, the SCPL patients died, but the TL patients did not; in the other 22 discordant pairs, the TL patients died, but the SCPL patients did not. There was no significant difference in the risk of overall death between the two treatment groups (hazard risk $[\mathrm{HR}]=1.15 ; 95 \% \mathrm{CI}$ : 0.79-1.67; $P=0.47$; Table 3).

In the 17 concordant pairs, both the SCPL and TL patients died from the disease. While in the 14 discordant pairs, the SCPL patients died from the disease, but the TL patients did not; and in the other 20 discordant pairs, the TL patients died

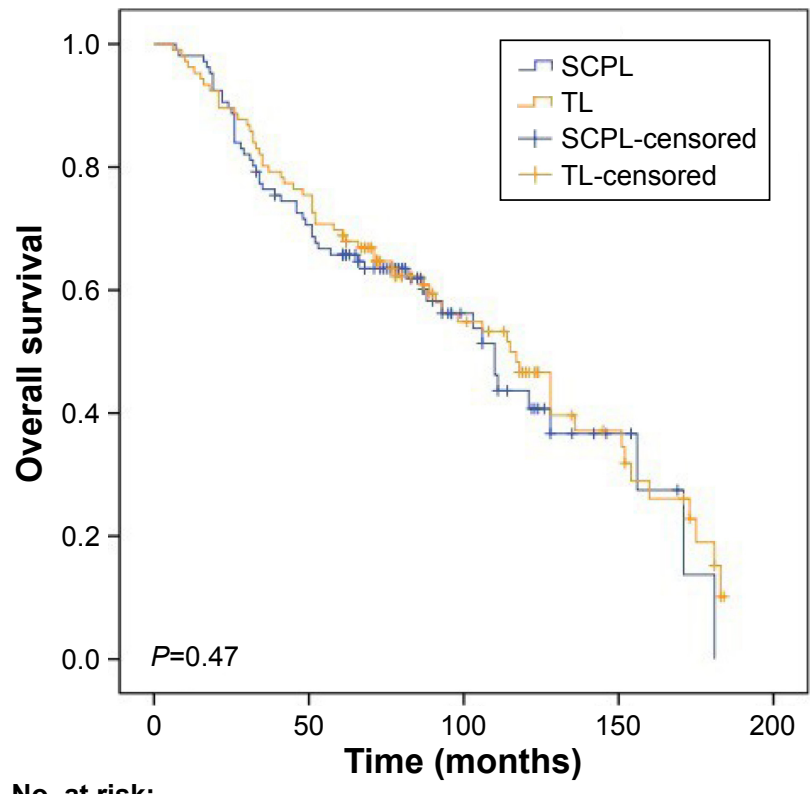

No. at risk:

$\begin{array}{llllc}\text { SCPL } & 106 & 73 & 23 & 5 \\ \text { TL } & 106 & 80 & 36 & 14\end{array}$

Figure I Comparison of the Kaplan-Meier survival curves on OS rate in the SCPL and $T L$ groups (log-rank $P=0.47$ ).

Note: Censoring is indicated by tick marks.

Abbreviations: OS, overall survival; SCPL, supracricoid partial laryngectomy; TL, total laryngectomy.

from the disease, but the SCPL patients did not. There was no significant difference in risk of death caused by the disease (HR=1.11; 95\% CI: $0.69-1.80 ; P=0.66$; Table 3 ).

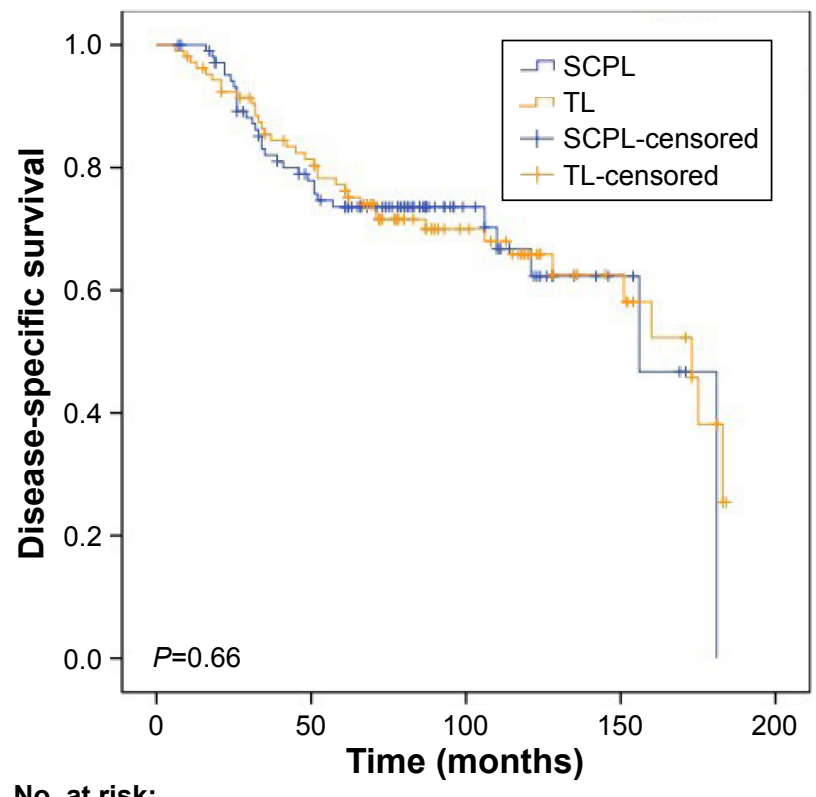

No. at risk:

$\begin{array}{llllc}\text { SCPL } & 106 & 73 & 23 & 5 \\ \text { TL } & 106 & 80 & 36 & 14\end{array}$

Figure 2 Comparison of the Kaplan-Meier survival curves on DSS rate in the SCPL and TL groups (log-rank $P=0.66$ ).

Note: Censoring is indicated by tick marks.

Abbreviations: DSS, disease-specific survival; SCPL, supracricoid partial laryngectomy; TL, total laryngectomy. 




No. at risk:

$\begin{array}{llllc}\text { SCPL } & 106 & 69 & 21 & 5 \\ \text { TL } & 106 & 73 & 35 & 12\end{array}$

Figure 3 Comparison of the Kaplan-Meier survival curves on RFS rate in the SCPL and $T L$ groups (log-rank $P=0.77$ ).

Note: Censoring is indicated by tick marks.

Abbreviations: RFS, recurrence-free survival; SCPL, supracricoid partial laryngectomy; TL, total laryngectomy.

Similarly, both the SCPL and TL patients exhibited disease recurrence in the 18 concordant pairs; while in the 19 discordant pairs, the SCPL patients exhibited disease recurrence, but the TL patients did not; and in the other 24 discordant pairs, the TL patients exhibited disease recurrence, but the SCPL patients did not. Again, no evidence of a significant difference in disease recurrence between these two groups was found (HR=1.07; 95\% CI: $0.68-1.68$; $P=0.77$; Table 3). Furthermore, multivariate analysis was performed for the unmatched factors, including comorbidity score, alcohol status, nodal classification, and differentiation grade, and there were no significant differences in OS, DSS, and RFS between the SCPL and TL groups (Table 3).

\section{Discussion}

Optimal treatment for the patients with $\mathrm{T} 3$ laryngeal carcinoma remains controversial. According to the recommendations by the National Comprehensive Cancer Network (NCCN), radiotherapy (with or without chemotherapy for organ preservation) is one of the treatment options. In addition, surgical management, including TL, continues to be useful for the patients who are not suitable for chemoradiotherapy, either due to the tumor characteristics (bulky tumors or tumors combined with cartilage destruction) or the patients' general or socioeconomic conditions. ${ }^{10}$ Considering a permanent tracheostoma and loss of natural voice resulted from organ's loss following TL surgery; organ-protective surgeries are important for the patients with T3 LSCC. Therefore, SCPL has recently been widely used at an increasing number of centers to improve quality of life in certain patients with advanced laryngeal cancer. ${ }^{12}$

SCPL was first described by Majer and Rieder ${ }^{13}$ in 1959 and was modified by Piquet et $\mathrm{al}^{14}$ in 1974 . Since then, numerous studies have demonstrated that reliable therapeutic and acceptable functional results have been archived using this procedure. Especially, SCPL has widely been used in Europe for the tumors involving the glottis or supraglottis without extra-laryngeal extension staged as $\mathrm{T} 3$ in order to preserve the laryngeal function. ${ }^{15-18}$

The goal of the surgery for LSCC is to completely remove the tumor while maximizing the retention or reconstruction of the pronunciation, breathing, and swallowing functions, ${ }^{19-21}$ thus, the surgical approach has been evolving from TL to organ preservation. A study conducted by Weinstein et $\mathrm{al}^{22}$ reported that the quality of life in patients with SCPL was superior to that of patients who underwent TL with tracheoesophageal puncture and that the histological assessment of whole organ sections of TL specimens indicated that many patients who had been subjected to TL might have been candidates for SCPL.

Few studies have reported to compare the outcomes between SCPL and TL in T3 LSCC, although the oncological results of SCPL as a primary treatment have been documented in the literature. ${ }^{23,24}$ Lima et al have reported a 5 -year DSS of 78\% and a 5-year RFS of $83 \%$ in 43 patients treated with SCPL-CHEP. ${ }^{25}$ Mercante et al ${ }^{18}$ have reported a 5-year disease-free survival and OS of $78.2 \%$ and $87.3 \%$, respectively, in T3 LSCC. In another study of 83 cases by De Virgilio et al, when TL was compared with SCPL-CHP among cT3 patients, a 3 -year OS of $82 \%$ vs $64 \%$ and a DSS

Table 3 Risks associated with different surgical procedures

\begin{tabular}{|c|c|c|c|c|c|c|}
\hline \multirow[b]{2}{*}{ Matched-pair analysis } & \multicolumn{3}{|c|}{ Risk, SCPL to TL } & \multicolumn{3}{|c|}{ Adjusted risk, SCPL to TL } \\
\hline & HR & $P$-value & $95 \% \mathrm{Cl}$ & HR & $P$-value & $95 \% \mathrm{Cl}$ \\
\hline Death, all cause & 1.15 & 0.47 & $0.79-1.67$ & 1.31 & 0.18 & $0.89-1.95$ \\
\hline Death, due to a disease & I.II & 0.66 & $0.69-1.80$ & 1.36 & 0.24 & $0.8 \mathrm{I}-2.28$ \\
\hline Recurrence & 1.07 & 0.77 & $0.68-1.68$ & 1.33 & 0.25 & $0.82-2.15$ \\
\hline
\end{tabular}

Abbreviations: SCPL, supracricoid partial laryngectomy; TL, total laryngectomy; HR, hazard risk. 
of $92 \%$ vs $73 \%$, respectively, were reported. ${ }^{12}$ Recently, Spriano et al reported that supracricoid laryngectomy by a lateral approach is feasible and safe, ${ }^{18}$ and most recently, Mannelli et al performed meta-analysis on the outcomes of two conservative treatments for advanced T3-T4 laryngeal cancer. ${ }^{26}$ They reported that the oncologic outcomes of transoral laser and open partial laryngectomies for advanced T3-T4 squamous cell laryngeal cancers were similar. ${ }^{26}$

In the current study, we matched 106 pairs of patients, who underwent SCPL or TL, using the matching variables of age, sex, smoking status, tumor site, disease stage, and adjuvant treatment. All cases were strictly limited to those with endolaryngeal invasive squamous cell carcinoma classified as T3 and were eligible for either treatment modalities with a minimum of 5 years of follow-up or longer up to death. Patients were informed on potential advantages and disadvantages of the two different treatment options, and treatment selection was mainly based on the patients' general condition and their subjective preferences. Patients with severe COPD and impairment of the cough reflex were considered unsuitable for SCPL. To the best of our knowledge, this matchedpair analysis was the only study thus far that compared TL and SCPL as primary surgical treatments in terms of survival in patients with LSCC classified as T3.

In the TL group, 5-year OS and DSS rates were 69.8\% and $77.2 \%$, respectively. As expected, comparable results (65.8\% and $73.6 \%$, respectively) were also achieved in the SCPL treatment group, as evidenced by the absence of significant differences between the two groups. In fact, SCPL resulted in slightly better 5-year RFS rates than TL $(72.1 \%$ vs $71.6 \%$, respectively), but no significant difference was found between the two groups. These findings were similar to those reported in the previous studies, although the rates of the current study were slightly lower. Potential reason of the difference in the rates might be that regional advanced stage (N2-N3) cases were predominated in this study.

It has been consistently reported that $\mathrm{N}$ stage is the most predictive characteristic of survival. ${ }^{27}$ Owing to the risk of occult nodal metastasis in T3, all the patients of this study received neck dissection and no patient experienced regional failure or complications related to neck dissection. This procedural policy allowed us to document the exact number of affected lymph nodes and the presence of extracapsular nodal spread and enabled us to choose the best adjuvant treatment for each patient. As a result, the percentage of pathological nodes that were classified as N2-N3 stage was $46.2 \%$, and we believed that this might be due to the high proportion $(38.7 \%)$ of supraglottic cancer, which was included in this study and theoretically had a higher incidence of lymph node metastasis.

Possibility of the bias for patient selection was one of the limitations of this study as it was a single hospital-based retrospective study. Second, a relatively small sample size (106 pairs) was also a potential limitation. Nevertheless, given the OS and follow-up times of this study, we had $80 \%$ power for detecting a minimal 1.6-fold increased risk of recurrence or death in the study groups. Finally, other potentially important data, such as human papillomavirus status, which were recently documented as a predictor of laryngeal cancer $^{28}$ and socioeconomic or marital status were not included in this study. These remained to be collected in the future larger studies to further validate the current findings.

\section{Conclusion}

No evidence of significant differences in OS, DSS, or RFS between the two surgical procedures of SCPL and TL used in the T3 LSCC patients was found when they were match paired by age, sex, smoking status, tumor site, disease stage, and adjuvant treatment. Findings of the current study suggested that SCPL has reliable therapeutic and functional outcomes for locally advanced tumors and might be used to avoid a TL in selected patients with advanced stage primary laryngeal cancer.

\section{Abbreviations}

AJCC, American Joint Committee on Cancer; CHEP, cricohyoidoepiglottopexy; CHP, cricohyoidopexy; DSS, disease-specific survival; HR, hazard risk; LSCC, laryngeal squamous cell carcinoma; NCCN, National Comprehensive Cancer Network; OS, overall survival; RFS, recurrence-free survival; SCPL, supracricoid partial laryngectomy; TL, total laryngectomy.

\section{Disclosure}

The authors report no conflicts of interest in this work.

\section{References}

1. Cancer IAFRO. GLOBOCAN 2012: Estimated Cancer Incidence, Mortality and Prevalence Worldwide in 2012. 2012.

2. Chen W, Zheng R, Baade PD, et al. Cancer statistics in China, 2015. CA Cancer J Clin. 2016;66(2):115-132.

3. Groome PA, O'Sullivan B, Irish JC, et al. Management and outcome differences in supraglottic cancer between Ontario, Canada, and the Surveillance, Epidemiology, and End Results areas of the United States. J Clin Oncol. 2003;21(3):496-505.

4. Luo J, Wu J, Lv K, et al. Analysis of Postsurgical Health-Related Quality of Life and Quality of Voice of Patients With Laryngeal Carcinoma. Medicine. 2016;95(1):e2363. 
5. Soudry E, Marmor Y, Hazan A, Marx S, Sadov R, Feinmesser R. Supracricoid partial laryngectomy: an alternative to total laryngectomy for locally advanced laryngeal cancers. J Laryngol Otol. 2008;122(11): 1219-1223.

6. Laccourreye O, Brasnu D, Biacabe B, Hans S, Seckin S, Weinstein G. Neo-adjuvant chemotherapy and supracricoid partial laryngectomy with cricohyoidopexy for advanced endolaryngeal carcinoma classified as T3-T4: 5-year oncologic results. Head Neck. 1998;20(7):595-599.

7. Karasalihoglu AR, Yagiz R, Tas A, Uzun C, Adali MK, Koten M. Supracricoid partial laryngectomy with cricohyoidopexy and cricohyoidoepiglottopexy: functional and oncological results. J Laryngol Otol. 2004;118(9):671-675.

8. Chen P, Yu W, Huang J, et al. Matched-pair analysis of survival in patients with poorly differentiated versus well-differentiated glottic squamous cell carcinoma. Oncotarget. 2017;8(9):14770-14776.

9. Kierzek A, Paprocka-Borowicz M, Pozowski A, Kuciel-Lewandowska J. The first Polish total laryngectomies. Contemp Oncol. 2013;17(6): 473-476.

10. Rodrigo JP, López F, Llorente JL, Álvarez-Marcos C, Suárez C. Results of total laryngectomy as treatment for locally advanced laryngeal cancer in the organ-preservation era. Acta Otorrinolaringol Esp. 2015;66(3):132-138.

11. Pytynia KB, Grant JR, Etzel CJ, Roberts DB, Wei Q, Sturgis EM. Matched-pair analysis of survival of never smokers and ever smokers with squamous cell carcinoma of the head and neck. J Clin Oncol. 2004;22(19):3981-3988.

12. de Virgilio A, Fusconi M, Gallo A, et al. The oncologic radicality of supracricoid partial laryngectomy with cricohyoidopexy in the treatment of advanced N0-N1 laryngeal squamous cell carcinoma. Laryngoscope. 2012;122(4):826-833.

13. Majer EH, Rieder W. Technic of laryngectomy permitting the conservation of respiratory permeability (cricohyoidopexy). Ann Otolaryngol. 1959;76:677-681.

14. Piquet JJ, Desaulty A, Decroix G. Crico-hyoido-epiglotto-pexy. Surgical technic and functional results. Ann Otolaryngol Chir Cervicofac. 1974;91(12):681-686.

15. Laccourreye H, Laccourreye O, Weinstein G, Menard M, Brasnu D. Supracricoid laryngectomy with cricohyoidopexy: a partial laryngeal procedure for selected supraglottic and transglottic carcinomas. Laryngoscope. 1990;100(7):735-741.

16. Rizzotto G, Crosetti E, Lucioni M, Succo G. Subtotal laryngectomy: outcomes of 469 patients and proposal of a comprehensive and simplified classification of surgical procedures. Eur Arch Otorhinolaryngol. 2012;269(6):1635-1646.
17. Gallo A, Manciocco V, Simonelli M, Pagliuca G, D’Arcangelo E, de Vincentiis M. Supracricoid partial laryngectomy in the treatment of laryngeal cancer: univariate and multivariate analysis of prognostic factors. Arch Otolaryngol Head Neck Surg. 2005;131(7):620-625.

18. Mercante G, Grammatica A, Battaglia P, Cristalli G, Pellini R, Spriano G. Supracricoid partial laryngectomy in the management of $\mathrm{t} 3$ laryngeal cancer. Otolaryngol Head Neck Surg. 2013;149(5):714-720.

19. Zhang SY, Lu ZM, Chen LS, et al. Supracricoid partial laryngectomy cricohyoidoepiglottopexy (SCPL-CHEP) versus vertical partial laryngectomy for the treatment of glottic carcinoma. Eur Arch Otorhinolaryngol. 2013;270(3):1027-1034.

20. Bron L, Brossard E, Monnier P, Pasche P. Supracricoid partial laryngectomy with cricohyoidoepiglottopexy and cricohyoidopexy for glottic and supraglottic carcinomas. Laryngoscope. 2000;110(4):627-634.

21. Castro A, Sanchez-Cuadrado I, Bernaldez R, del Palacio A, Gavilan J. Laryngeal function preservation following supracricoid partial laryngectomy. Head Neck. 2012;34(2):162-167.

22. Weinstein GS, El-Sawy MM, Ruiz C, et al. Laryngeal preservation with supracricoid partial laryngectomy results in improved quality of life when compared with total laryngectomy. Laryngoscope. 2001;111(2): 191-199.

23. Farrag TY, Koch WM, Cummings CW, et al. Supracricoid laryngectomy outcomes: The Johns Hopkins experience. Laryngoscope. 2007; 117(1):129-132.

24. Ozturk K, Akyildiz S, Gode S, et al. Post-Surgical and Oncologic Outcomes of Supracricoid Partial Laryngectomy: A Single-Institution Report of Ninety Cases. ORL J Otorhinolaryngol Relat Spec. 2016;78(2): 86-93.

25. Lima RA, Freitas EQ, Dias FL, et al. Supracricoid laryngectomy with cricohyoidoepiglottopexy for advanced glottic cancer. Head Neck. 2006;28(6):481-486.

26. Mannelli G, Lazio MS, Luparello P, Gallo O. Conservative treatment for advanced T3-T4 laryngeal cancer: meta-analysis of key oncological outcomes. Eur Arch Otorhinolaryngol. 2018;275(1):27-38.

27. Matsuo JM, Patel SG, Singh B, et al. Clinical nodal stage is an independently significant predictor of distant failure in patients with squamous cell carcinoma of the larynx. Ann Surg. 2003;238(3):106-116 discussion 412-421.

28. Chen X, Gao L, Sturgis EM, et al. HPV16 DNA and integration in normal and malignant epithelium: implications for the etiology of laryngeal squamous cell carcinoma. Ann Oncol. 2017;28(5):1105-1110.
OncoTargets and Therapy

\section{Publish your work in this journal}

OncoTargets and Therapy is an international, peer-reviewed, open access journal focusing on the pathological basis of all cancers, potential targets for therapy and treatment protocols employed to improve the management of cancer patients. The journal also focuses on the impact of management programs and new therapeutic agents and protocols on

\section{Dovepress}

patient perspectives such as quality of life, adherence and satisfaction. The manuscript management system is completely online and includes a very quick and fair peer-review system, which is all easy to use. Visit http://www.dovepress.com/testimonials.php to read real quotes from published authors. 